\title{
Development of Open Source Tools that support the CCOG Resolution
}

\author{
Jim Sutherland
}

Key words: CCOG, national georeferencing initiative, requirements, infrastructure

\section{National Geo-Referencing Initiative}

The Canadian Council on Geomatics (CCOG) passed a resolution in March 2005 whereby each province/territory agreed to develop and implement a plan to require geo-referencing new legal surveys according to the principles and standards set out in the resolution. Michael O'Sullivan, former Surveyor General of Canada, initiated the resolution which took over five years of research, development, analysis and discussion before being passed.

The resolution sets out the responsibility to each province/territory to define three georeferencing zones. The zones are to be defined as a function of parcel location and land use.

The resolution also sets out the required minimum relative accuracy standard, at the $95 \%$ confidence level, for geo-referencing new legal surveys located within each zone as follows:

$\begin{array}{ll}\text { Survey location } & \text { Accuracy } 95 \% \text { Confidence Level } \\ \text { Urban areas } & 5 \mathrm{~cm} \\ \text { Rural areas } & 20 \mathrm{~cm} \\ \text { Remote areas } & 1 \text { metre }\end{array}$

The referencing datum must be NAD83 (CSRS) and ties, where reasonable, shall be made to high quality officially published High Precision Networks (HPN), Canadian Base Net (CBN) or Active Control Points (ACP). Currently in BC there are HPN networks in the Capital Regional District and Greater Vancouver Regional District areas, approximately a dozen CBN pillars, and approximately eighteen BC-ACP GPS reference stations.

A fundamental principle of the resolution is that requirements (legislation, regulations, rules) for integration of new legal surveys, to the standards, should only be implemented when there is sufficient and easy access to the reference framework.

This leaves the provinces and territories to develop and implement a plan to:

1. Assess their local geo-referencing infrastructure and enhance where necessary to ensure access is practical.

2. Communicate the migration strategy for mandatory geo-referencing to the survey community.

3. Solicit input from land surveyors for defining geo-referencing zones.

4. Assess land surveyors training requirements and bridge gaps where necessary.

5. Explore the opportunity for collaborating on the development of open source tools that will facilitate and support geo-referencing new legal surveys nationally.

6. Make necessary legislation, regulation or rule changes. 


\section{BC Geo-Referencing Requirements}

The current General Survey Instruction Rules require all legal surveys outside of an integrated survey area, conducted all or in part by GPS methods to be geo-referenced to two (2) metres or better at the $95 \%$ confidence level. All legal surveys within integrated survey areas must be geo-referenced to $5 \mathrm{~cm}$ or better at the $95 \%$ confidence level. There is also a fairly recent rule change that requires Mineral Tenure Act surveys to be geo-referenced to 0.5 metres or better at $95 \%$ confidence level.

A working group, chaired by Mike Taylor, was created about a year and a half ago by the Government Liaison Committee (GLC) to investigate technical aspects of geo-referencing legal surveys and the practicality of accessing the positioning infrastructure within BC. The summary report of the working group findings on geo-referencing legal surveys was presented to the members at the last annual general meeting. The report includes an excellent summary of available positioning methods and accuracies achievable.

In alignment with the CCOG resolution for integration of legal surveys passed in March 2005, the GLC has taken the follow-up initiative to develop draft rule changes to require mandatory geo-referencing of all legal surveys in BC. The rule change will be presented to the Survey Rules Committee (SRC) in the near future. In support of this proposed rule change, the GLC is sponsoring a workshop at the Annual General Meeting which is intended to provide a good overview of positioning technologies and specifically outline the practicality of geo-referencing legal surveys in BC.

\section{Geo-Referencing Infrastructure}

The provinces, territories and Natural Resources Canada (NRCan) have worked together to modernize the geo-referencing framework across Canada for many years. This collaborative effort has resulted in the readjustment of regional geodetic control networks and the official adoption of the NAD83 (CSRS) datum. The federal leadership and regional cooperation to enhance geo-spatial reference system has provided a much more homogenous network of geodetic control across Canada. Diagram 1 illustrates the hierarchy of the national geodetic control and regional control in British Columbia.

The philosophies and resources available within the provincial and territorial areas to develop the regional geodetic referencing framework over the last many years resulted in quite a variance in the physical and active control infrastructure developed within each region. As Brad Hlasny, Manager of the Geo-Spatial Reference Section, Base Mapping and Geomatics Services Branch (BMGS) said in the March 2004 issue of The Link, "Truly, we are lucky to live in B.C. when it comes to accessing a modern and effective geo-spatial reference system (with its associated positioning services and tools)". It is important to note that BMGS is actively pursuing cooperative partnerships with local, regional governments and educational institutes to further enhance the provincial active control infrastructure. New active control points are in the process of being set up in Castlegar and Prince George with other areas currently under discussion and consideration.

One recent advance in the geo-referencing environment is the online Precise Point Positioning (PPP) Service launched by NRCan (Federal Government). This service enables users with 


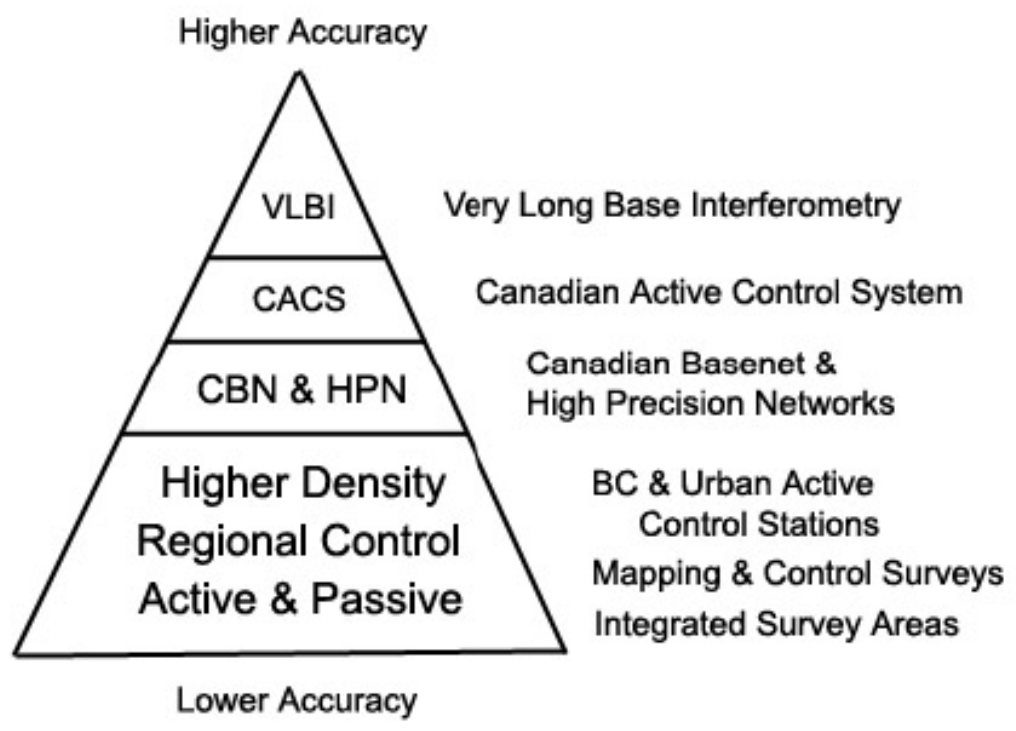

Figure 1: Accuracy

one receiver to derive reasonably accurate NAD83 (CSRS) positions relative to the Canadian Active Control System (CACS). The user collects GPS data at a point, exports a file in the RINEX file format, and then submits this file to the online PPP service. The on-line process is very fast and the user is emailed results and comprehensive processing reports. The processing can also be performed offline as well by using GPS Pace software.

The improved accuracy of the positioning solution is derived from precise GPS orbit and clock information which is collaboratively collected and distributed by agencies participating in the International GPS Service. Single or dual frequency receivers may be used, however, Precise Point Positioning using dual frequency receivers takes advantage of using both the carrier phase and pseudo-range observations. The PPP processing for single frequency receiver's currently uses only the pseudo-range observations. As well, only dual frequency receivers can take advantage of direct measurement of atmospheric errors. Single frequency receivers must instead rely on modelling these errors with a result of reduced positional accuracy.

Base Mapping and Geomatics Services Branch is currently testing the PPP service using data collected with various receivers including low cost single frequency receivers. The tests are expected to include typical legal survey observation scenarios (see diagram 2) that include PPP derived coordinates, $3 \mathrm{D}$ vectors (derived from differential positioning using two receivers) and conventional direction/distance observations. I believe the PPP service will have a great impact in making it practical and relatively inexpensive for land surveyors to geo-reference new legal surveys in remote areas.

The availability of inexpensive new generation GPS receivers that are capable of collecting multi-channel carrier phase and pseudo-range data and able to convert to a RINEX file format will complement the PPP service to provide a powerful and practical positioning solution. New processing techniques and technologies also show promise for providing better positioning solutions using inexpensive GPS receivers, thereby, making high accuracy positioning more practical/accessible to the survey community. 
Diagram 2

Legal Survey Network

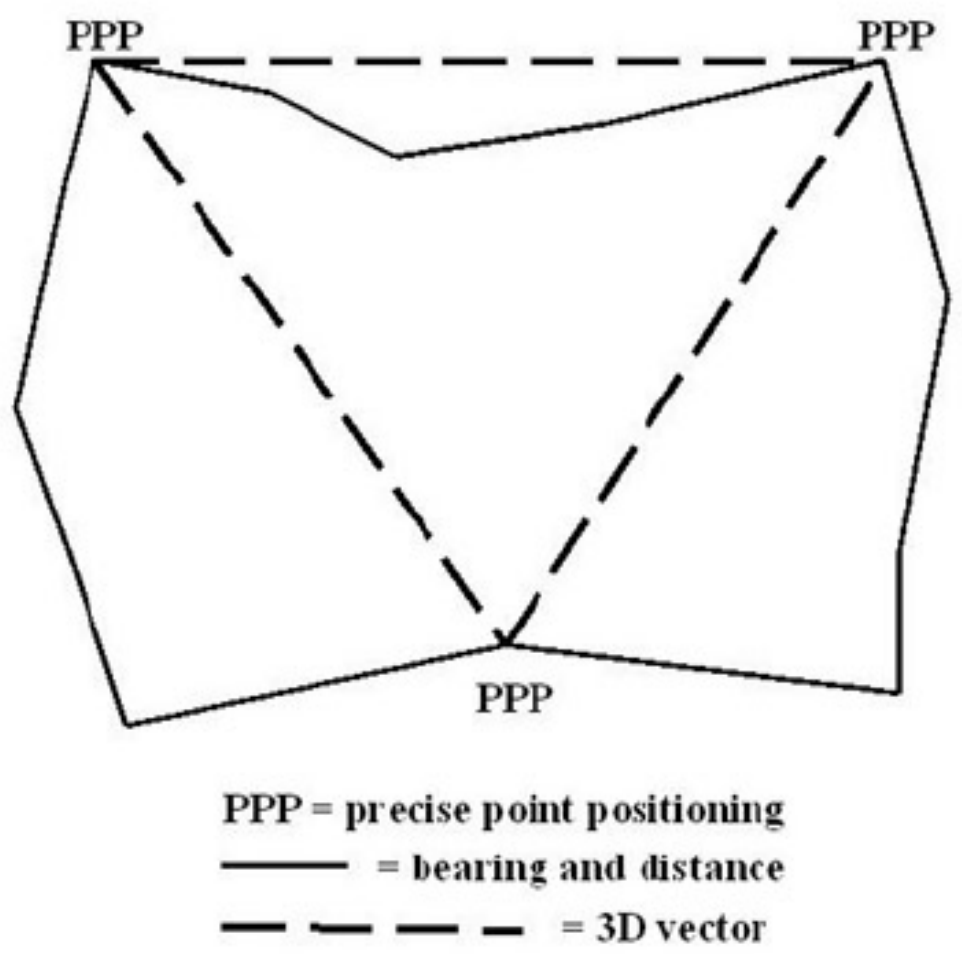

Figure 2: Legal Survey Network

\section{Integrating Legal Surveys}

The challenge facing the legal survey community is to determine whether the geodetic framework is adequate in each region to implement rules for mandatory geo-referencing of all new legal surveys, and if not, how to enhance it to be practical. The cost of ramping-up all legal survey firms to be capable of geo-referencing all new legal surveys could be considerable. One must look at not only the capital cost of survey equipment, but also the equipment validation, training, maintenance and processing resources.

Alternatively, many survey firms have already taken advantage of modernizing survey operations to include global positioning systems in order to gain efficiencies and to embark on new business opportunities. While the implementation of geo-referencing of all surveys will involve additional work not previously undertaken during legal surveys in many areas of the Province, the long term benefits to the cadastre and to surveyors can not be understated.

\section{Development of Open Source Tools that Support the CCOG Resolution}

The resolution passed by CCOG fosters the integration of legal surveys across Canada. However, this places an additional burden on land surveyors to find practical methods for georeferencing and integrating their conventional legal surveys to meet the standards. It would 
be very beneficial to all stakeholders to collectively explore the development of an integrated set of open source tools for processing conventional survey and coordinate observations. This would provide an effective alternative to commercial software that would benefit the national survey community.

We are very fortunate to have a number of existing open source tools that provide valuable resources independently, however, when combined and enhanced would provide a very powerful and practical processing environment for land surveyors. A brief outline of some of pertinent existing open source programs follows:

Gama Least Square Adjustment is a program for performing a least square adjustment of 1D, 2D and 3D survey observations. The project was initiated by Ales Cepek and Jan Pytel at the Department of Mapping and Cartography, Faculty of Civil Engineering, Czech Technical University in Prague in 1998. The program name Gama is derived as an acronym from Geodesy and Mapping. Gama has been presented at FIG conferences and received status of GNU license software in 2001. Gama adjusts observed coordinates (with variance- covariance matrix), distances, angles/directions, height differences and $3 \mathrm{D}$ vectors in a local coordinate system. The observation data is formatted as an XML (Extensible Markup Language) input file. This makes it easy to read and edit the data. Gama is run simply as a command line program or via the companion GUI Rocinante (written by Jan Pytel) which is very well structured and easy to use.

Rocinante Graphical User Interface for Gama is fully object-oriented cross-platform GUI for creating/editing Gama XML input files and running Gama least square program.

Use-Friendly Desktop GIS (uDig) is an open-source spatial data viewer/editor based on OpenGIS standards and is licensed under Lesser Gnu Public License (LGPL) and includes a Web Map Server and Web Feature Server. uDig provides a common Java platform for building spatial applications with open source components.

GeoTools is an open source Java GIS toolkit for developing programs compliant with Open Geospatial Consortium standards. GeoTools provides a computational process of converting a position given in one Coordinate Reference System into the corresponding position in another Coordinate Reference System.

Gama and Rocinante are very powerful survey data processing resources for land surveyors. They were originally intended for adjusting geodetic networks, however, Rocinante makes it easy to input legal survey type observations and quickly run the Gama least square adjustment. Land surveyors commonly use commercial coordinate geometry programs to process their conventional direction and distance survey observations. In recent years some firms have incorporated GPS base line processors and complimentary least square adjustment programs that may or may not include processing both conventional and GPS data together.

Many commercial tools enable land surveyors to export an AutoCAD TM DXF format file. This provides an opportunity to use this file format as an easy means of importing the direction and distance observations to the Gama XML input format. uDig provides an excellent framework for creating a plug-in extension to import the DXF file, convert to Gama XML input format and run the adjustment. UDig can also perform necessary coordinate transforms using the appropriate GeoTools code. Adding a plug-in extension to import coordinate obser- 
vations (including the associated variance co-variance matrix) from Precise Point Positioning files would create a powerful and practical integrated legal survey observations processing environment.

Diagram 3 illustrates a basic proposed legal survey data processing model integrating the aforementioned open source tools. The model enables a user to:

1. create observation input file using Rocinante;

2. input conventional observations using a vector file input (DXF / SHP) and extract to Gama (note: the vector file would be coded by line-type or colour for discerning direction, distance or both type of observations);

3. input coordinate observations via PPP, text file or dialogue input;

4. input Land XML file format observations and convert to Gama XML input format;

5. perform coordinate transforms;

6. run Gama least square adjustment, review output, edit and re-run as necessary;

7. export final adjusted network in DXF, SHP, Land XML format or Gama output file.

Creating a robust and easy to use set of integrated legal survey open source processing tools will provide substantial support to land surveyors in all regions tasked with the requirement of geo-referencing legal surveys according to the new CCOG resolution. We are very fortunate to have all the main open source components already existing to create an integrated processing environment that will require minimal additional effort.

The March 2005 CCOG resolution set the stage for the migration towards mandatory georeferencing of legal surveys across Canada. I strongly encourage the national survey community to seek support from CCOG and the Geo-Connections program in order to collectively explore, develop and implement a powerful integrated open source processing solution for integration of legal surveys.

\section{References}

1. Gama Least Square Program ${ }^{1}$

2. Rocinante GUI for Gama ${ }^{2}$

3. uDig Home Page ${ }^{3}$

4. GeoTools Home Page ${ }^{4}$

5. GeoConnections ${ }^{5}$

6. Canadian Council on Geomatics (CCOG) ${ }^{6}$

\footnotetext{
${ }^{1}$ http://www.gnu.org/software/gama/

${ }^{2}$ http://roci.sourceforge.net/

${ }^{3}$ http://udig.refractions.net/confluence/display/UDIG/Home

${ }^{4}$ http://www.geotools.org/

${ }^{5}$ http://www.geoconnections.org/CGDI.cfm/fuseaction/partners.ccog/gcs.cfm

${ }^{6}$ http://www.cgdi.gc.ca/ccog/ccog.html
} 


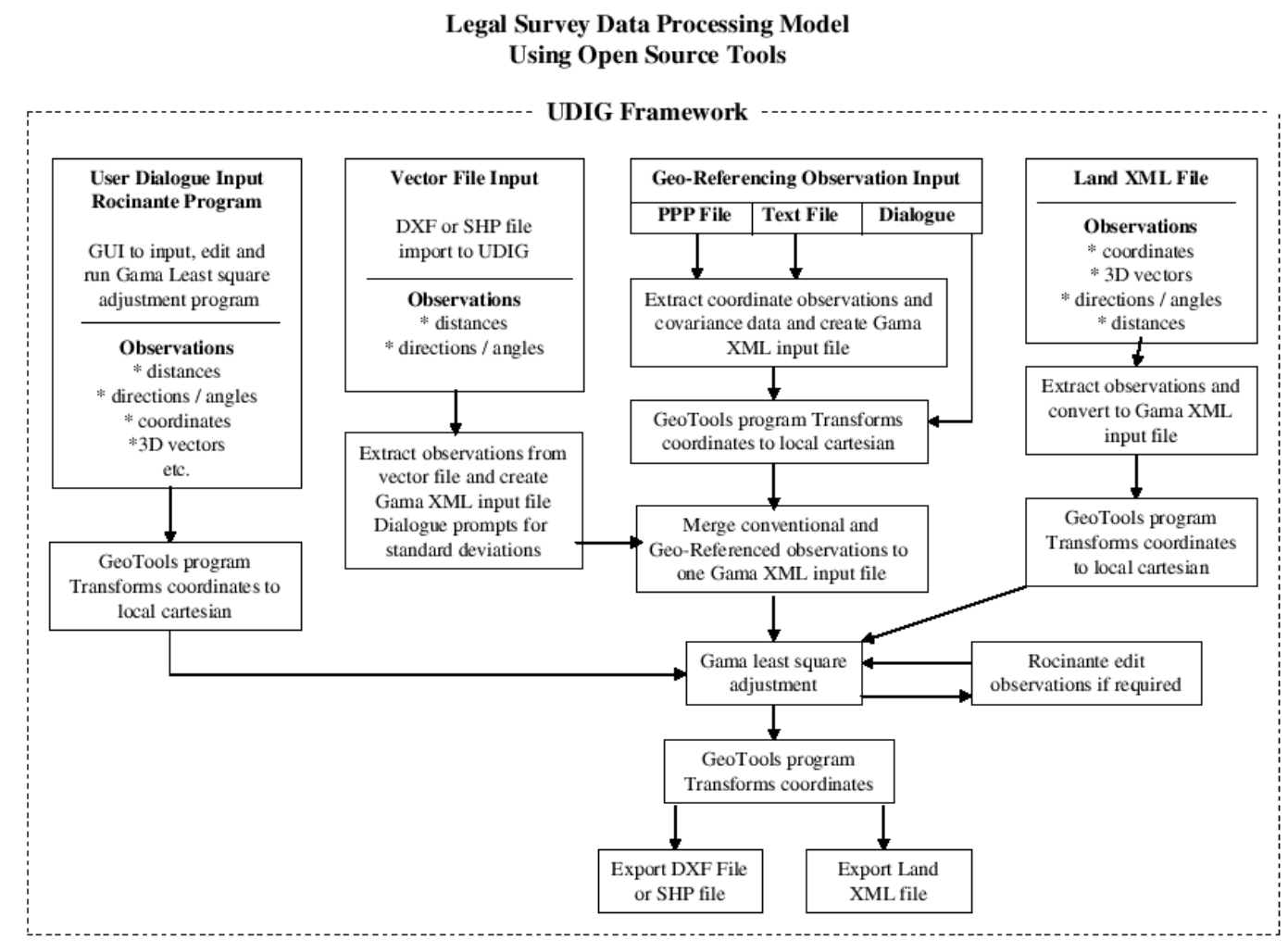

Figure 3: UDIG Framework

7. Precise Point Positioning Service ${ }^{7}$

8. Canadian Spatial Reference System ${ }^{8}$

9. British Columbia Geo Spatial Reference System ${ }^{9}$

\footnotetext{
${ }^{7}$ http://www.geod.nrcan.gc.ca/ppp_e.php

${ }^{8}$ http://www.geod.nrcan.gc.ca/network_lp/index_e.php

${ }^{9}$ http://srmwww.gov.bc.ca/bmgs/gsr/
} 